\title{
On Linear Resolution
}

Sam Buss*

Department of Mathematics

University of California, San Diego

La Jolla, CA 92093-0112, USA

Jan Johannsen ${ }^{\dagger}$

Institut für Informatik

Ludwig-Maximilians-Universität München

80538 München, Germany sbuss@ucsd.edu

jan.johannsen@ifi.lmu.de

\begin{abstract}
We discuss the relationship between linear resolution, s-linear resolution and other fragments of resolution, including tree-like resolution, regular resolution and general resolution. We also discuss linear resolution with restarts. We present polynomial-size linear resolution proofs of the ordering tautologies (also known as "graph tautologies"), and the guarded ordering tautologies. This shows that regular resolution does not simulate linear resolution.
\end{abstract}

KEYWORDS: s-resolution, linear resolution, tree-like resolution, regular resolution, proof complexity

\section{Introduction}

Resolution is a propositional proof system which underlies the most successful methods for theorem proving and satisfiability (SAT) testing used by present-day SAT solvers. Resolution is so successful not because it is a particularly strong proof system, but rather because it supports efficient strategies for searching for proofs or refutations. Accordingly, there has been extensive work, going back to the 1960's, on refinements of resolution that allow more efficient search strategies.

This paper examines the complexity of one such refinement, linear resolution, which was introduced independently by Loveland [20], Luckham [21] and Zamov and Sharonov [31] as a refined form of resolution that might allow more efficient search strategies. Linear resolution is not used in present-day SAT solvers however. Instead, the most successful SAT solvers use conflict-driven clause learning (CDCL), i.e., variants of DPLL algorithms with clause learning $[22,3]$. Nonetheless, linear resolution is still of considerable interest. In terms of proof complexity, linear resolution lies between tree-like and general resolution. It is known that tree-like resolution does not (polynomially) simulate general resolution or linear resolution, though the latter had been an open problem for a long time and was only recently shown [11]. However, it is open whether linear resolution simulates general resolution.

* Supported in part by NSF grant CCR-1213151. Work performed in part at the Special Semester on Complexity Theory at St. Petersburg State University, and Dagstuhl seminar 16381 "SAT and Interactions".

$\dagger$ Work performed in part during a visit at UCSD, the Special Semester on Complexity Theory at St. Petersburg State University, and Dagstuhl seminar 16381 "SAT and Interactions". 
These questions have been considered before, and there have been several incorrect proofs of such simulations and non-simulations circulated as preprints and even appearing in the literature. In particular, Mayr [23] claimed that tree-like resolution and linear resolution are p-equivalent (this is false); Bonet-Galesi [8] gave an erroneous proof that the ordering (graph) tautologies $\operatorname{Ord}_{n}$ have polynomial size linear resolution refutations (this is now established below as Theorem 14), which implies an exponential separation between tree-like and linear resolution; and a retracted preprint of Buresh-Oppenheim-Mitchell-Pitassi [10] claimed an exponential separation between linear resolution and full and regular resolution (this is still open).

There are a number of intriguing parallels between CDCL and linear resolution. ${ }^{1 .}$ Even without restarts, both systems (polynomially) simulate tree-like resolution, but are not simulated by tree-like resolution (for linear resolution, see [11] or Corollary 15 below). It is also known that regular resolution does not simulate either CDCL or linear resolution, even for the systems without restarts (for CDCL, see [1]; for linear resolution, see Corollary 18 of the present paper). For both systems when restarts are disallowed, it is unknown whether they simulate general resolution. Indeed, when restarts are disallowed, both systems have the property that they are "closed under restrictions" (see Definition 11) if and only if they simulate general resolution (see [3] for CDCL, and Theorem 12 and [26] for linear resolution). Finally, both systems have the property that when restarts are allowed, they do simulate resolution (see [3, 2, 24] for CDCL, and Theorem 8 and [26] for linear resolution).

On the other hand, for a "non-parallel", it is essentially trivial that (non-greedy) CDCL without restarts simulates regular resolution, but it is open whether linear resolution also simulates regular resolution. It is also open whether either of CDCL without restarts or linear resolution simulates the other.

Section 2 defines linear resolution and a restricted form called s-linear resolution, and discusses their known relationships to tree-like and general resolution. Section 3 has our main results. First, we give short linear resolution refutations of the ordering principles. This establishes a direct proof that tree-like resolution does not simulate linear resolution. Second, Theorem 17 and Corollary 18 extend these results to the guarded ordering tautologies to show that also regular resolution does not simulate linear resolution.

We presume the reader is familiar with the basic concepts of satisfiability, such as "variable", "literal", "clause", "resolution inference", "resolution refutation", "tree-like", "dag-like" etc. For these, consult [3] for instance. We assume always that clauses are non-tautological; that is, no clause contains both $x$ and $\bar{x}$ for $x$ a literal.

Acknowledgement. We thank Alasdair Urquhart for suggesting the problem of linear resolution to us. We also thank the referees for useful comments and suggestions.

1. Knowledge of CDCL is not needed for the present paper, but the reader unfamiliar with CDCL may consult $[3,5]$ for more information. When we talk about "CDCL without restarts", we mean the nongreedy versions which can ignore conflicts. This can be formulated as a proof system either in terms of pool resolution [29] or in terms of resolution trees with lemmas, i.e. the system regWRTI [12]. All the comments in the introduction about "CDCL without restarts" should be interpreted as applying to both pool resolution and regWRTI. 


\section{Linear versus tree-like versus general resolution}

We let $\Gamma$ denote a set of clauses.

Definition 1. A linear resolution derivation of a clause $C$ from $\Gamma$ is a sequence of clauses $C_{1}, \ldots, C_{m}$ such that

a. $C_{1}$ is a clause from $\Gamma$,

b. $C_{m}$ is the clause $C$, and

c. For every $i<m, C_{i+1}$ is the resolvent of $C_{i}$ either with a clause $D$ from $\Gamma$ or with a clause $C_{k}$ for some $k<i$.

If $C$ is the empty clause $\emptyset$, then this is called a linear resolution refutation of $\Gamma$. When the second condition of c. holds, we call $C_{i}$ the near parent of $C_{i+1}$, and $C_{k}$ the far parent of $C_{i+1}$, and we say that there is a backarc from $i+1$ to $k$. When the first condition of c. holds, the clause $D$ is called an input clause.

Some papers, including [10, 11, 25], state an incorrect definition of linear resolution, in which the third clause of the definition is replaced by

c. For every $i<m, C_{i+1}$ is (1) the resolvent of $C_{i}$ with a clause $D$ from $\Gamma$, (2) the resolvent of $C_{i}$ with a clause $C_{k}$ for some $k<i$, or (3) a member of $\Gamma$.

(Although the definition is misstated in the papers [11, 25], the proofs in those papers still apply to the correctly defined version of linear resolution, so the theorems in $[11,25]$ about linear resolution are indeed correct.) We call the system thus defined linear resolution with restarts. Linear resolution with restarts is equivalent to general resolution; this was first shown by Rachinsky [26], and is reproved below as Theorem 8 .

Loveland [20] and Zamov and Sharonov [31] defined the following refined notion of "slinear resolution", where a backarc is admissible only if the conclusion is a subclause of the near parent. (The terminology is from [20]; "s" stands for subsumption.)

Definition 2. An s-linear resolution derivation of $C$ from $\Gamma$ is a sequence of clauses $C_{1}, \ldots, C_{m}$ such that (a) $C_{1} \in \Gamma$, (b) $C_{m}$ is $C$; and

(c) For every $i<m, C_{i+1}$ is either

- the resolvent of $C_{i}$ with a clause $D$ from $\Gamma$, or

- the resolvent of $C_{i}$ with a clause $C_{k}$ for some $k<i$ such that $C_{i+1} \subseteq C_{i}$.

The condition " $C_{i+1} \subseteq C_{i}$ " is called the subsumption condition. It is not known whether s-linear resolution simulates linear resolution (in the sense of Definition 4 below).

A general resolution derivation $P$ from $\Gamma$ can be naturally represented as a finite directed acyclic graph: the nodes of the graph are the occurrences of clauses in $P$. The source nodes (indegree zero) are initial clauses from $\Gamma$. Every other clause has indegree two, and is inferred by resolution from its two parent clauses. The graph has a single sink, labelled with the conclusion clause. If every clause other than the conclusion clause has outdegree 1 , then $P$ is tree-like. 
Definition 3. A derivation $P$ is regular provided that it does not contain any irregularity, i.e., a directed path in the graph for $P$ from a clause inferred by resolution on a literal $x$ to another clause which contains $x$ or $\bar{x}$.

Definition 4. Let $\mathcal{R}_{1}$ and $\mathcal{R}_{2}$ be refinements of resolution. We say that $\mathcal{R}_{1}$ simulates $\mathcal{R}_{2}$ if every $\mathcal{R}_{2}$ derivation of $n$ inferences deriving a clause $C$ from $\Gamma$ can be converted into a $\mathcal{R}_{1}$ derivation with $n^{O(1)}$ inferences deriving a clause $C^{\prime} \subseteq C$ from $\Gamma$. We say that $\mathcal{R}_{1}$ p-simulates $\mathcal{R}_{2}$ if this conversion can be carried out by a polynomial time procedure. $\mathcal{R}_{1}$ and $\mathcal{R}_{2}$ are (p-)equivalent if they (p-)simulate each other.

Remark: The notions "simulate" and "p-simulate" are usually defined using only refutations rather than derivations; namely, taking $C$ to be the empty clause. Our definition is slightly more general, and potentially stronger. Our later results about non-simulation (Corollaries 15 and 18) hold also for the more traditional definition of "simulate".

The next theorem is from [20] and is implicit in [31]; see also [17], [30], [19] and [26, Thm 4.1].

Theorem 5. S-linear resolution p-simulates tree-like resolution.

As a consequence, (s-)linear resolution is complete; that is, if $\Gamma \vDash C$, then there is an s-linear resolution derivation of some clause $C^{\prime} \subseteq C$ from $\Gamma$.

Proof. Suppose that $P$ is a tree-like resolution derivation of the clause $D$ from $\Gamma$ with $n$ inferences, and that $C$ is a $\Gamma$-clause which is used in $P$. Without loss of generality, $P$ is regular. We shall prove there is an s-linear resolution derivation $P^{\prime}$ of $D$ from $\Gamma$ with $\leq 2 n$ inferences such that $C$ is the first clause in $P^{\prime}$. The proof is by induction on $n$. The base case $n=0$ is trivial. So suppose $n \geq 1$, and $P$ ends with a resolution inference

$$
\frac{A, x \quad B, \bar{x}}{A, B}
$$

Here it is understood that $x \notin A$ and $\bar{x} \notin B$, and that $A, B$ denotes the union of $A$ and $B$. Let $P_{1}$ and $P_{2}$ denote the (disjoint) subproofs of $P$ ending with $A, x$ and $B, \bar{x}$, respectively. By regularity, no clause in $P_{1}$ contains $\bar{x}$ and no clause in $P_{2}$ contains $x$. Also, no clause in $P$ contains the complement of any literal in $A, B$.

W.l.o.g, $C$ is a leaf of $P_{1}$. By the induction hypothesis, there is a linear resolution derivation $C_{1}=C, C_{2}, C_{3}, \ldots, C_{m}$ from $\Gamma$ with $C_{m}$ equal to $A, x$. Choose $D_{1}$ to be any $\Gamma$ clause used in $P_{2}$ such that $\bar{x} \in D_{1}$. The induction hypothesis gives an s-linear derivation $P_{2}^{\prime}$ from $\Gamma$ of the form $D_{1}, D_{2}, \ldots, D_{\ell}$ with $D_{\ell}$ equal to $B, \bar{x}$. We modify $P_{2}^{\prime}$ to form a linear resolution derivation $D_{1}^{\prime}, D_{2}^{\prime}, \ldots, D_{\ell}^{\prime}$ where $D_{1}^{\prime}$ is equal to $A, D_{1} \backslash\{\bar{x}\}$ and where $D_{\ell}^{\prime}$ is either $A, B$ or $A, B, \bar{x}$. Specifically, we define $D_{i}^{\prime}$ to equal $A, D_{i}$ if for some $j \leq i$, the clause $D_{j}$ is inferred in $P_{2}^{\prime}$ by resolution with a $\Gamma$-clause containing $\bar{x}$, and define $D_{i}$ equal to $A, D_{i} \backslash\{\bar{x}\}$ if no such $j$ exists. Thus, $P_{2}^{\prime}$ has exactly the same structure as $P_{2}$, and uses exactly the same input parents as $P_{2}$, except that $D_{1}$ is replaced with $D_{1}^{\prime}$, and the changes in $D_{1}$ are propagated down through $P_{2}$ to form $P_{2}^{\prime}$. It is easy to check that $D_{1}^{\prime}, D_{2}^{\prime}, \ldots, D_{\ell}^{\prime}$ still satisfies the subsumption condition. 
Let $E$ be the clause $A, B$. The clause $D_{\ell}^{\prime}$ is equal to either $A, B$ or to $A, B, \bar{x}$. In the latter case, the desired refutation $P^{\prime}$ is defined to be

$$
C_{1}, C_{2}, \ldots, C_{m}, D_{1}^{\prime}, D_{2}^{\prime}, \ldots, D_{\ell}^{\prime}, E \text {. }
$$

Recall that $C_{m}$ is the clause $A, x$. The clause $D_{1}^{\prime}$ is derived by resolution from $C_{m}$ and the $\Gamma$-clause $D_{1}$ w.r.t. $x$. And, the clause $E$ is derived by resolving $C_{m}$ and $D_{\ell}^{\prime}$, so there is a backarc from $E$ to $C_{m}$. The other clauses are inferred as in $P_{1}^{\prime}$ and $P_{2}^{\prime}$. Thus, $P^{\prime}$ is a linear resolution refutation. Furthermore, the inference giving $E$ clearly satisfies the subsumption principle, so $P^{\prime}$ is an s-linear resolution refutation. In the case where $D_{\ell}^{\prime}$ equals $A, B$, the s-linear resolution refutation $P^{\prime}$ is defined in the same way, except the final clause $E$ is omitted (and thus no backarc is added).

As a side remark, the proof of Theorem 5 can be modified to show that "t-linear resolution" p-simulates tree-like resolution; The definition of t-linear resolution is omitted here as it is rather technical to state; it was introduced by Kowalski and Kuehner [17] as a restricted version of s-linear resolution that might be more amenable to proof search than (s-)linear resolution.

Definition 6. A crossing in a linear resolution derivation $P$ is defined to be four indices $i_{1}>i_{2}>j_{1}>j_{2}$ such that $P$ contains backarcs from $i_{1}$ to $j_{1}$ and from $i_{2}$ to $j_{2}$.

Inspection of the proof of Theorem 5 shows that the constructed s-linear derivation contains no crossings, therefore tree-like resolution is p-simulated by s-linear resolution without crossings, and hence by linear resolution without crossings.

We show now that the opposite p-simulation works as well, so linear resolution without crossings is p-equivalent to tree-like resolution.

Theorem 7. Tree-like resolution p-simulates linear resolution without crossings.

Proof. We show by induction on $n$ that any crossing-free linear resolution derivation of size $n$ can be simulated by a tree-like resolution derivation of size at most $n$.

Suppose that $D$ is derived from $\Gamma$ by a linear resolution derivation $P$ without crossings, and that $P$ has size $n$. Without loss of generality, the final inference is a backarc, i.e., $D=A, B$ is derived from its near parent $A, x$ and its far parent $B, \bar{x}$. Since the derivation has no crossings, it consists of a linear derivation $P_{1}$ without crossings of $B, \bar{x}$ from $\Gamma$ of size $n_{1}<n$, followed by a linear derivation without crossings $P_{2}$ of $A, x$ from $\Gamma \cup\{B, \bar{x}\}$ of size $n_{2}<n$, plus the final inference, so that $n=n_{1}+n_{2}+1$.

By the induction hypothesis, there is a tree-like derivation $T_{1}$ of size at most $n_{1}$, of some clause $B^{\dagger} \subseteq B, \bar{x}$. If $B^{\dagger} \subseteq B$, then we are done, otherwise we have $B^{\dagger}$ equal to $B^{\prime}, \bar{x}$ for some $B^{\prime} \subseteq B$.

By the induction hypothesis again, there is a tree-like derivation $T_{2}$ of size at most $n_{2}$, of some clause $A^{\dagger} \subseteq A, x$ from $\Gamma \cup\{B, \bar{x}\}$. Consider any occurrence of $B, \bar{x}$ as an initial clause in $T_{2}$. Since $\bar{x}$ does not appear in $A^{\dagger}$, there must be a last clause $C, \bar{x}$ on the path from $B, \bar{x}$ to $A^{\dagger}$ that contains $\bar{x}$. This clause $C, \bar{x}$ is resolved in $T_{2}$ against a clause $C^{\prime}, x$ yielding $C, C^{\prime}$. The subtree deriving $C, \bar{x}$ is pruned from $T_{2}$, and every clause $E$ on the path from $C, C^{\prime}$ to $A^{\dagger}$ is replaced by some clause $E^{\prime} \subseteq E, x$. Repeating this for every leaf in $T_{2}$ 
labelled $B, \bar{x}$ yields a tree-like derivation $T_{2}^{\prime}$ of some clause $A^{\ddagger} \subseteq A^{\dagger}, x$ from $\Gamma$. Clearly $T_{2}$ has size at most $n_{2}$.

Again, if $A^{\ddagger} \subseteq A$, we are done, otherwise we have $A^{\ddagger}=A^{\prime}, x$ for some $A^{\prime} \subseteq A$, and we join $T_{1}$ and $T_{2}^{\prime}$ with a resolution inference obtaining $A^{\prime}, B^{\prime} \subseteq A, B$. The resulting tree-like derivation is of size at most $n_{1}+n_{2}+1=n$.

The system of linear resolution with restarts was defined after Definition 1. We now show it is equivalent to general resolution, as was first shown by Rachinsky [26].

Theorem 8. (Rachinsky [26]) Linear resolution with restarts p-simulates general resolution.

Proof. Let $P$ be a resolution derivation of $C$ from $\Gamma$, and let $C_{1}, \ldots, C_{m}$ be a topological ordering of the clauses in $P$. By induction on $k$, we show that there is a linear derivation with restarts $P_{k}$ deriving $C_{k}$ from $\Gamma$ such that $P_{k}$ has at most $k^{2}$ steps and contains all the clauses $C_{1}, \ldots, C_{k}$. The induction basis is trivial since $C_{1} \in \Gamma$ : the derivation $P_{1}$ consists of $C_{1}$ alone.

For the induction step, let $C_{k}$ be derived from $C_{i}$ and $C_{j}$ with $i, j<k$. The derivation $P_{k-1}$ contains $C_{j}$. Now let $E_{1}, \ldots, E_{\ell}$ be a path in $P$ leading from an initial clause $E_{1} \in \Gamma$ to $E_{\ell}=C_{i}$. Each clause $E_{r+1}$ on that path is derived from $E_{r}$ and some clause $E$ that occurs in $P_{k-1}$. Therefore the sequence obtained by appending $E_{1}, \ldots, E_{\ell}, C_{k}$ to $P_{k-1}$ is a linear derivation $P_{k}$ with restarts as required. The number of inferences in $P_{k}$ is at most $(k-1)^{2}+\ell+1 \leq(k-1)^{2}+(k-1)+1<k^{2}$.

The final $P_{m}$ is a linear derivation with restarts of $C_{m}$ from $\Gamma$ with at most $m^{2}$ inferences.

The next definition is a simplified version of a construction from Rachinsky [26].

Definition 9. Let $\Gamma$ be a set of clauses and $y$ be a variable not appearing in $\Gamma$. The set of clauses $\Gamma^{* y}$ is defined to be the set of clauses in $\Gamma$ plus the clauses

- $a \vee y$ for every literal $a$ appearing in $\Gamma$,

- $C \vee \bar{y}$ for every clause $C$ in $\Gamma$.

Proposition 10. If $C$ has a resolution derivation from $\Gamma$ with $n$ inferences, then $C$ has a linear resolution refutation from $\Gamma^{* y}$ with $O\left(n^{3}\right)$ inferences.

Proof. By Theorem 8, there is a linear resolution derivation with restarts $P$ of $C$ from $\Gamma$ of at most $n^{2}$ inferences. Let the clauses in $P$ be $C_{1}, \ldots, C_{\ell}$, with $\ell \leq n^{2}$ and $C_{\ell}=C$. We show how to transform $P$ into a linear resolution derivation.

Let $X$ be the set of literals resolved on in $P$. Since the original derivation had $n$ inferences, $|X| \leq n$. Let $Y=C \backslash X$; this is the set of literals in $C$ not resolved on in $P$. If $x \in Y$, then its complement $\bar{x}$ does not appear anywhere in $P$. We shall define clauses $C_{i}^{\prime}$, for $i=1, \ldots, n$ such that each $C_{i} \subseteq C_{i}^{\prime} \subseteq C_{i} \cup Y$ and such that $C_{1}^{\prime}, \ldots, C_{\ell}^{\prime}$ can be pieced together to form a valid linear derivation $P^{\prime}$ from $\Gamma^{* y}$. The final clause $C_{\ell}^{\prime}$ equals $C$ since $C_{\ell} \subseteq C_{\ell}^{\prime} \subseteq C_{\ell} \cup Y=C_{\ell}$.

The clause $C_{1}^{\prime}$ is just $C_{1}$. If $C_{i+1}$ is inferred in $P$ by resolution from $C_{i}$ and $D$ where $D \in \Gamma$, then let $C_{i+1}^{\prime}$ equal the resolvent of $C_{i}^{\prime}$ and $D$. Similarly, if $C_{i+1}$ is inferred from $C_{i}$ 
and $C_{k}$, then let $C_{i+1}^{\prime}$ equal the resolvent of $C_{i}^{\prime}$ and $C_{k}^{\prime}$. The non-trivial case is when $C_{i+1}$ is a restart in $P$, i.e., $C_{i+1} \in \Gamma$. Let

$$
\left\{a_{1}, a_{2}, \ldots, a_{k}\right\}:=C_{i} \backslash Y=C_{i}^{\prime} \backslash Y=C_{i} \cap X=C_{i}^{\prime} \cap X .
$$

Between $C_{i}$ and $C_{i+1}$ we insert resolution inferences with the $\Gamma^{* y}$-clauses $\bar{a}_{j} \vee y$ for $1 \leq j \leq k$, giving the clause $\left(C_{i}^{\prime} \cap Y\right), y$, and then resolve against the $\Gamma^{* y}$-clause $C_{i+1} \vee \bar{y}$ to obtain $C_{i+1}^{\prime}$.

The clauses $C_{1}^{\prime}, \ldots, C_{n}^{\prime}$ plus the clauses inserted to handle restarts form valid linear resolution refutation $P^{\prime}$. Since the number $k$ of extra inferences inserted between $C_{i}^{\prime}$ and $C_{i+1}^{\prime}$ is at most $n+1$, the number of resolution inferences in $P^{\prime}$ is $O\left(n^{3}\right)$.

A result similar to Proposition 10 was proved by [11] using a different, more complex formula "AddTaut $(\Gamma)$ ". Both our construction in Proposition 10 and the one in [11] show that linear resolution effectively p-simulates general resolution in the sense of [25]. It is open whether Proposition 10 can be improved to give a polynomial size s-linear resolution refutation.

For $\rho$ a restriction (a partial truth assignment) and $C$ a clause, we define $C \uparrow \rho$ as follows: If $\rho(x)=$ True for some $x \in C$, then $C \uparrow \rho=$ True; otherwise $C \uparrow \rho$ is $C$ minus the literals set False by $\rho$. For $\Gamma$ a set of clauses, $\Gamma\lceil\rho$ is the set of clauses $C\lceil\rho$ for $C \in \Gamma$ such that $C \uparrow \rho \neq$ True.

Definition 11. A refinement $\mathcal{R}$ of resolution is closed under restrictions if whenever there is a $\mathcal{R}$-derivation of a clause $C$ from $\Gamma$ with $n$ inferences and whenever $\rho$ is a restriction such that $C\left\lceil\rho \neq\right.$ True, then there is a $\mathcal{R}$-refutation of a clause $C^{\prime} \subseteq C \uparrow \rho$ from $\Gamma \uparrow \rho$ with $n^{O(1)}$ inferences.

Analogously to our earlier remark about "simulate" and "p-simulate", our definition of "closed under restrictions" is a little stronger than the usual definition which considers only refutations (that is, with $C$ and $C^{\prime}$ both equal to the empty clause).

The following observation is also due to Rachinsky [26]:

Theorem 12. Linear resolution is closed under restrictions iff it p-simulates general resolution.

Proof. The "if" part is obvious since general resolution is closed under restrictions. For the converse, suppose $C$ has a resolution derivation from $\Gamma$ of size $n$. Then by Proposition 10, $C$ has a linear resolution derivation from $\Gamma^{* y}$ of polynomial size in $n$. The restriction setting the new variable $y$ to True restores $\Gamma$ from $\Gamma^{* y}$. Also, $C\lceil\rho$ is the same as $C$ since $y$ does not appear in $C$. Thus, if linear resolution is closed under restrictions, there is a linear resolution derivation of some clause $C^{\prime} \subseteq C$ from $\Gamma$ of polynomial size.

Since tree-like and regular resolution are closed under restrictions, this construction also provides separations of these resolution refinements from linear resolution. We can give two examples. First, Bonet and Galesi [9] showed that the ordering principle tautologies $\operatorname{Ord}_{n}$ (which we define below) provide an exponential separation between tree-like resolution and general resolution. Hence, since tree-like resolution is closed under restrictions, the " $* y$-translations" $\left(\operatorname{Ord}_{n}\right)^{* y}$ also require exponential size tree-like resolution refutations. 
Therefore the $\left(\operatorname{Ord}_{n}\right)^{* y}$ principles provide an exponential separation between tree-like resolution and linear resolution. Second, Alekhnovich et al. [1] gave exponential lower bounds on regular resolution refutations for the guarded ordering principle tautologies (these are also defined below), so by the same argument their $* y$-translations provide an exponential separation between regular resolution and linear resolution.

Buresh-Oppenheim and Pitassi [11, Lemma 4.5] earlier gave similar separations for these and other systems using the related AddTaut translation.

These separations based on $* y$ - and AddTaut-translations are somewhat artificial, and the next section gives more natural, direct separations.

\section{The ordering principle in linear resolution}

The ordering principle encapsulates the fact that a totally ordered finite set has a largest element. This was first studied by Krishnamurthy [18] as a potential hard example for resolution, but Stålmarck [28] showed that it has short regular resolution refutations. Nevertheless, its propositional proof complexity has been further studied by many authors $[9,14,13,27,4,29,1,15,7,16]$. Our propositional translations of the ordering principle use the variables $x_{i, j}$ with $1 \leq i, j \leq n$ and $i \neq j$. The variable $x_{i, j}$ is intended to express that $i \prec j$ in a linear ordering $\prec$. We will thus adopt the simplifying convention of $[6,7]$ that $x_{i, j}$ and $\bar{x}_{j, i}$ are the same literal, i.e., only the variables $x_{i, j}$ for $i<j$ actually exist, and $x_{j, i}$ for $j>i$ is just a notation for $\bar{x}_{i, j}$, so that $\bar{x}_{j, i}$ stands for $x_{i, j}$. This identification makes no essential difference to the complexity of resolution refutations of these formulas, but it reduces the number of literals and clauses, and simplifies the definitions. In particular, it means there are no axioms for the antisymmetry or totality of $\prec$.

Definition 13. Fix $n \geq 1$. The ordering principle $\operatorname{Ord}_{n}$ is the following set of clauses:

1. The maximality clauses $M_{i}^{(n)}=\bigvee_{j \in\{1, \ldots, n\} \backslash\{i\}} x_{i, j}$, for each $1 \leq i \leq n$.

2. The transitivity clauses $T_{i, j, k}:=\bar{x}_{i, j} \vee \bar{x}_{j, k} \vee \bar{x}_{k, i}$ for all distinct $i, j, k \in\{1, \ldots, n\}$.

The maximality clause $M_{i}^{(n)}$ states that $x$ is not an $\prec$-maximal element. The transitivity axiom $T_{i, j, k}$ states that if $i \prec j$ and $j \prec k$, then $i \prec k$.

Bonet and Galesi [9] gave an exponential lower bound for tree-like resolution refutations of $\operatorname{Ord}_{n}$. (They call them "graph tautologies $\mathrm{GT}_{n}$ instead of $\operatorname{Ord}_{n}$.) Thus the following theorem gives a direct separation between tree-like and linear resolution. (This theorem was stated earlier in [8]; however, the part of their proof that applied to linear resolution contained an error, and was therefore omitted from the journal version [9].)

Theorem 14. There are linear resolution refutations of the ordering principle $\mathrm{Ord}_{n}$ with $O\left(n^{3}\right)$ inferences.

Corollary 15. Tree-like resolution does not simulate linear resolution.

Proof. The general idea of the proof is to derive the $\operatorname{Ord}_{n-1}$ clauses from $\operatorname{Ord}_{n}$, and then iterate until deriving the $\mathrm{Ord}_{2}$ clauses. The $\mathrm{Ord}_{2}$ principle has just the two unit clauses $\left\{x_{1,2}\right\}$ and $\left\{x_{2,1}\right\}$, so a single additional inference gives the empty clause. Our main construction gives a linear resolution derivation from $\operatorname{Ord}_{n}$ of size $O\left(n^{2}\right)$ which starts with the 


$$
\frac{\bigvee_{j \in \mathcal{J}} x_{n, j} \vee \bigvee_{j \in\{1, \ldots, n-1\} \backslash\{i\}} x_{i, j} \quad \bar{x}_{n, j_{0}}, \bar{x}_{j_{0}, i}, \bar{x}_{i, n}}{\frac{\bigvee_{j \in \mathcal{J}^{\prime}} x_{n, j} \vee \bigvee_{j \in\{1, \ldots, n-1\} \backslash\{i\}} x_{i, j} \vee \bar{x}_{i, n}}{\bigvee_{j \in \mathcal{J}^{\prime}} x_{n, j} \vee \bigvee_{j \in\{1, \ldots, n-1\} \backslash\{i\}} x_{i, j}} \bigvee_{j \in\{1, \ldots, n\} \backslash\{i\}} x_{i, j}}
$$

Figure 1. The construction used for the induction step of Lemma 16. The three clauses on the left are $C[\mathcal{J}] \vee M_{i}^{(n-1)}$ and $C\left[\mathcal{J}^{\prime}\right] \vee M_{i}^{(n-1)} \vee \bar{x}_{i, n}$ and $C\left[\mathcal{J}^{\prime}\right] \vee M_{i}^{(n-1)}$. They are resolved against $T_{n, j_{0}, i}$ and $M_{i}^{(n)}$. The clause $\mathcal{J}^{\prime}$ is $\mathcal{J} \backslash\left\{j_{0}\right\}$.

clause $M_{n}^{(n)}$ and ends with $M_{n-1}^{(n-1)}$. This derivation will contain the clauses $M_{i}^{(n-1)}$ for every $i \leq n-1$. It will consist of

- First, a linear resolution derivation of $M_{1}^{(n-1)}$ from $\operatorname{Ord}_{n}$ which starts with $M_{n}^{(n)}$. This contains $O(n)$ resolution inferences.

- Second, linear resolution derivations from $\operatorname{Ord}_{n} \cup\left\{M_{i}^{(n-1)}\right\}$ which start with $M_{i}^{(n-1)}$ and end with $M_{i+1}^{(n-1)}$, one for each $i \leq n-1$. Each of these contains $O(n)$ resolution inferences.

Iterating these constructions gives a linear resolution refutation of $\operatorname{Ord}_{n}$ with $O\left(n^{3}\right)$ resolution inferences.

We fix the value $n$, and describe the linear resolution derivation of the $\operatorname{Ord}_{n-1}$ clauses from $\operatorname{Ord}_{n}$. We use " $\mathcal{J}$ " to denote a subset of $\{1, \ldots, n-1\}$. The clause $C[\mathcal{J}]$ is defined to be

$$
C[\mathcal{J}]:=\bigvee_{j \in \mathcal{J}} x_{n, j}
$$

Note that $M_{n}^{(n)}$ is the same as $C[\mathcal{J}]$ for $\mathcal{J}=\{1, \ldots, n-1\}$. The proof of Theorem 14 will repeatedly use the following construction:

Lemma 16. Let $i<n$ and suppose $\mathcal{J} \subseteq\{1, \ldots, n-1\} \backslash\{i\}$. There is a linear resolution derivation with $O(|\mathcal{J}|)$ resolution inferences which starts with $C[\mathcal{J}] \vee M_{i}^{(n-1)}$ and ends with $M_{i}^{(n-1)}$ and uses only $M_{i}^{(n)}$ and transitivity clauses as input clauses.

Proof. We describe the refutation by recursion on $|\mathcal{J}|$. If $\mathcal{J}=\emptyset$, then $C[\mathcal{J}]$ is the empty clause and the lemma is trivial. Otherwise, let $j_{0} \in \mathcal{J}$ (say the least element of $\mathcal{J}$ ). Let $\mathcal{J}^{\prime}=\mathcal{J} \backslash\left\{j_{0}\right\}$. As shown in Figure 1, we derive $C\left[\mathcal{J}^{\prime}\right] \vee M_{i}^{(n-1)}$ from $C[\mathcal{J}] \vee M_{i}^{(n-1)}$ as follows. First, resolve $C[\mathcal{J}] \vee M_{i}^{(n-1)}$ and $T_{n, j_{0}, i}$ w.r.t. the resolution variable $x_{j_{0}, n}$; this yields the clause $C\left[\mathcal{J}^{\prime}\right] \vee M_{i}^{(n-1)} \vee \bar{x}_{i, n}$ since it removes $x_{n, j_{0}}$, adds $\bar{x}_{i, n}$, and leaves $x_{i, j_{0}}$ untouched since it is already in $M_{i}^{(n-1)}$. (By our conventions on literals, $T_{n, j_{0}, i}$ is the same $\left.\bar{x}_{n, j_{0}}, \bar{x}_{i, n}, x_{i, j_{0}}.\right)$ Then resolve $C\left[\mathcal{J}^{\prime}\right] \vee M_{i}^{(n-1)} \vee \bar{x}_{i, n}$ with $M_{i}^{(n)}$ w.r.t. $x_{i, n}$ to obtain $C\left[\mathcal{J}^{\prime}\right] \vee M_{i}^{(n-1)}$.

Iterating this construction until $\mathcal{J}=\emptyset$ proves the lemma. 
The derivation of $M_{1}^{(n-1)}$ from $\operatorname{Ord}_{n}$ is constructed as follows: First resolve $M_{n}^{(n)}$ and $M_{1}^{(n)}$ w.r.t. $x_{n, 1}$ to infer $C[\mathcal{J}] \vee M_{1}^{(n-1)}$ where $\mathcal{J}=\{2, \ldots, n-1\}$. Then apply the construction of Lemma 16 with $i=1$ to obtain $M_{1}^{(n-1)}$.

We now construct the derivation of $M_{i+1}^{(n-1)}$ from $M_{i}^{(n-1)}$. Define $D_{i}[j]$ to be the clause

$$
D_{i}[j]:=\bigvee_{k \in\{j, \ldots n-1\} \backslash\{i\}} x_{i, k} \vee \bigvee_{k \in\{1, \ldots n-1\} \backslash\{i\}} x_{n, k}
$$

Note that $D_{i}[i]$ is the same as $D_{i}[i+1]$. Also, $D_{i}[n]$ is the same as $M_{n}^{(n)}$ except lacking the literal $x_{n, i}$. As we describe next, the derivation of $M_{i+1}^{(n-1)}$ from $M_{i}^{(n-1)}$ first derives successively the clauses $D_{i}[j]$ for $j=2, \ldots, n$; it then derives $M_{i+1}^{(n-1)}$ from $D_{i}[n]$ with the aid of Lemma 16.

The first part starts by resolving $M_{i}^{(n-1)}$ and $T_{n, i, 1}$ w.r.t. $x_{i, 1}$ to obtain the clause $\bigvee_{j \in\{2, \ldots, n-1\} \backslash\{i\}} x_{i, j} \vee \bar{x}_{n, i} \vee x_{n, 1}$, and then resolving with $M_{n}^{(n)}$ w.r.t. $x_{i, n}$. This yields the clause $D_{i}[2]$. For successive values of $j=2, \ldots, i-1, i+1, \ldots, n-1$, the clause $D_{i}[j]$ is resolved with $T_{n, i, j}$ w.r.t. $x_{i, j}$ to obtain $D_{i}[j+1] \vee x_{n, j} \vee \bar{x}_{n, i}$ and this clause is resolved with $M_{n}^{(n)}$ w.r.t. $x_{i, n}$, yielding the clause $D_{i}[j+1]$.

Resolving $D_{i}[n]$ with $M_{i+1}^{(n)}$ w.r.t. the variable $x_{i+1, n}$ yields the clause $C[\mathcal{J}] \vee M_{i+1}^{(n-1)}$ where $\mathcal{J}=\{1, \ldots, n-1\} \backslash\{i, i+1\}$. Then, using Lemma 16 , we derive $M_{i+1}^{(n-1)}$.

This completes the construction of the linear resolution derivation of the $\operatorname{Ord}_{n-1}$ clauses from the $\operatorname{Ord}_{n}$ clauses. By inspection, the derivation has $O\left(n^{2}\right)$ steps. Putting these together gives the desired linear resolution derivation for Theorem 17.

The guarded ordering principle [1] (these are also called the "guarded graph tautologies") is obtained from $\operatorname{Ord}_{n}$ as follows: For each triple $i, j, k$ we associate distinct values $r=$ $r(i, j, k)$ and $s=s(i, j, k)$ such that $\{r, s\} \cap\{i, j, k\}=\emptyset$, and such that $r(i, j, k)=r(j, k, i)=$ $r(k, i, j)$ and similarly for $s$. Then each transitivity clause $T_{i, j, k}$ is replaced by two clauses $T_{i, j, k} \vee x_{r, s}$ and $T_{i, j, k} \vee \bar{x}_{r, s}$.

For the guarded ordering principle, an exponential lower bound for regular resolution refutations was shown by Alekhnovich et al. [1]. Thus the following provides a direct separation of regular and linear resolution.

Theorem 17. There are linear resolution refutations of the guarded ordering principle with $O\left(n^{3}\right)$ inferences.

Corollary 18. Regular resolution does not simulate linear resolution.

Of Theorem 1\%. We modify the linear resolution refutation of Theorem 14. Consider any use of a transitivity axiom $T_{i, j, k}$ in the refutation in Theorem 14, and let $r=r(i, j, k)$ and $s=s(i, j, k)$. W.l.o.g. (by renumbering subscripts), the original proof used a resolution inference on $C \vee x_{i, j}$ and $T_{i, j, k}$ w.r.t. $x_{i, j}$, giving $C \vee \bar{x}_{j, k} \vee \bar{x}_{k, i}$. If $C$ contains either $x_{r, s}$ or $\bar{x}_{r, s}$, replace this use of $T_{i, j, k}$ with $T_{i, j, k} \vee x_{r, s}$ or $T_{i, j, k} \vee \bar{x}_{r, s}$ (respectively). Otherwise replace this use of $T_{i, j, k}$ with three resolution inferences as pictured in Figure 2; first resolving $C \vee x_{i, j}$ with $T_{i, j, k} \vee x_{r, s}$ w.r.t. $x_{i, j}$, then resolving with $T_{i, j, k} \vee \bar{x}_{r, s}$ w.r.t. $x_{r, s}$, and finally resolving with $C \vee x_{i, j}$ w.r.t. $x_{i, j}$. Note that this creates a backarc to the clause $C \vee x_{i, j}$. This yields $C \vee \bar{x}_{j, k} \vee \bar{x}_{k, i}$ as desired. 


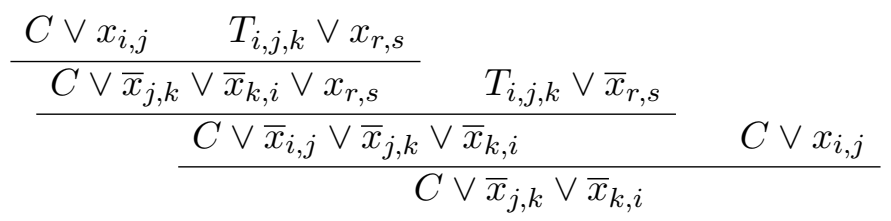

Figure 2. Resolving with guarded transitivity clauses for Theorem 17. The final resolution inference uses a backarc to the first clause.

We conclude with some open problems. First, the strength of s-linear resolution relative to either tree-like or regular resolution is unknown: Does tree-like resolution simulate slinear resolution? Do either of regular resolution or s-linear resolution simulate the other system? Note that Theorems 14 and 17 do not give s-linear resolution refutations. Second, does linear resolution simulate either pool resolution [29] or regWRTI [12]? Conversely, do either of the latter systems simulate linear resolution? Finally, does linear resolution simulate general resolution?

\section{References}

[1] Michael Alekhnovich, Jan Johannsen, Toniann Pitassi, and Alasdair Urquhart. An exponential separation between regular and general resolution. Theory of Computing, 3(5):81-102, 2007.

[2] Albert Atserias, Johannes Klaus Fichte, and Marc Thurley. Clause-learning algorithms with many restarts and bounded-width resolution. Journal of Artificial Intelligence Research, 40:353-373, 2011.

[3] Paul Beame, Henry A. Kautz, and Ashish Sabharwal. Towards understanding and harnessing the potential of clause learning. Journal of Artificial Intelligence Research, 22:319-351, 2004.

[4] Arnold Beckmann and Samuel R. Buss. Separation results for the size of constant-depth propositional proofs. Annals of Pure and Applied Logic, 136:30-55, 2005.

[5] Armin Biere, Marijn J.H. Heule, Hans van Maaren, and Toby Walsh, editors. Handbook of Satisfiability. Frontiers in Artificial Intelligence and Applications 185. IOS Press, Amsterdam, 2009.

[6] Maria Luisa Bonet and Samuel R. Buss. An improved separation of regular resolution from pool resolution and clause learning. In Proc. 15th International Conference on Theory and Applications of Satisfiability Testing - SAT 2012, Lecture Notes in Computer Science \#7317, pages 45-57, 2012.

[7] Maria Luisa Bonet, Samuel R. Buss, and Jan Johannsen. Improved separations of regular resolution from clause learning proof systems. Journal of Artificial Intelligence Research, 49:669-703, 2014. 
[8] Maria Luisa Bonet and Nicola Galesi. A study of proof search algorithms for resolution and polynomial calculus. In 40th Annual IEEE Symp. on Foundations of Computer Science, pages 422-431. IEEE Computer Society, 1999.

[9] Maria Luisa Bonet and Nicola Galesi. Optimality of size-width tradeoffs for resolution. Computational Complexity, 10(4):461-474, 2001.

[10] Josh Buresh-Oppenheim, David Mitchell, and Toniann Pitassi. Linear and negative resolution are weaker than resolution. Technical Report TR 01-074, Electronic Colloquium on Computational Complexity (ECCC), October 2001. Retracted in Comment 1, December 2002.

[11] Joshua Buresh-Oppenheim and Toniann Pitassi. The complexity of resolution refinements. Journal of Symbolic Logic, 72(4):1336-1352, 2007. Preliminary version in: Proc. 18th IEEE Symposium on Logic in Computer Science (LICS 2003).

[12] Samuel R. Buss, Jan Hoffmann, and Jan Johannsen. Resolution trees with lemmas: Resolution refinements that characterize DLL-algorithms with clause learning. Logical Methods in Computer Science, 4, 4:13(4:13):1-18, 2008.

[13] Yuval Filmus. On the Alekhnovich-Razborov degree lower bound for for the polynomial calculus. Unpublished manuscript, http://www.cs.toronto.edu/ỹuval/AlRa.pdf, 2014.

[14] Nicola Galesi and Massimo Lauria. Optimality of size-degree tradeoffs for polynomial calculus. ACM Transactions on Computational Logic, 12(1):Article 4, October 2010.

[15] Jan Johannsen. An exponential lower bound for width-restricted clause learning. In Proc. 12th International Conference on Theory and Applications of Satisfiability Testing - SAT 2009, Lecture Notes in Computer Science \#5584, pages 128-140, 2009.

[16] Alexander Knop. IPS-like proof systems based on binary decision diagrams. Typeset manuscript, June 2017.

[17] Robert Kowalski and Donald Kuehner. Linear resolution with selection function. Artificial Intelligence, 2:227-260, 1971.

[18] Balakrishnan Krishnamurthy. Short proofs for tricky formulas. Acta Informatica, 22(3):253-275, 1985.

[19] Reinhold Letz. First-Order Calculi and Proof Procedures for Automated Deduction. PhD thesis, Technische Hochschule, Darmstadt, July 1993.

[20] Donald W. Loveland. A linear format for resolution. In Symposium on Automated Deduction, Lecture Notes in Mathematics 125, pages 147-162. Springer Verlag, 1970.

[21] David Luckham. Refinement theorems in resolution theory. In Symposium on Automated Deduction, Lecture Notes in Mathematics 125, pages 163-191. Springer Verlag, 1970.

[22] João P. Marques-Silva and Karem A. Sakallah. GRASP - A new search algorithm for satisfiability. IEEE Transactions on Computers, 48(5):506-521, 1999. 
[23] Klaus Mayr. Refinements and extensions of model elimination. In Logic Programming and Automated Reasoning, LPAR'93, pages 217-228, Berlin, Heidelberg, 1993. Springer.

[24] Knot Pipatsrisawat and Adnan Darwiche. On the power of clause-learning SAT solvers as resolution engines. Artificial Intelligence, 172(2):512-525, 2011.

[25] Toniann Pitassi and Rahul Santhanam. Effectively polynomial simulations. In Innovations in Computer Science (ICS), pages 370-381, 2010.

[26] Nicolas Rachinsky. The complexity of resolution refinements and satisfiability algorithms. Diploma thesis, Ludwig-Maximilians Universität, Munich, 2008.

[27] Nathan Segerlind, Samuel R. Buss, and Russell Impagliazzo. A switching lemma for small restrictions and lower bounds for $k$-DNF resolution. SIAM Journal on Computing, 33(5):1171-1200, 2004.

[28] Gunnar Stålmarck. Short resolution proofs for a sequence of tricky formulas. Acta Informatica, 33(3):277-280, 1996.

[29] Allen Van Gelder. Pool resolution and its relation to regular resolution and DPLL with clause learning. In Logic for Programming, Artificial Intelligence, and Reasoning (LPAR 2005), Lecture Notes in Computer Science 3835, pages 580-594. SpringerVerlag, 2005.

[30] André Vellino. The Complexity of Automated Reasoning. PhD thesis, Dept. of Computer Science, University of Toronto, 1989.

[31] Nail K. Zamov and Vladislav I. Sharonov. A class of strategies for the determination of provability by the resolution method. In A.O. Slisenko, editor, Studies in Constructive Mathematics and Mathematical Logic, Part III, Seminars in Mathematics, V.A. Steklov Mathematical Institute, Leningrad, Volume 16, pages 26-31. Consultants Bureau, New York, 1971. Translated from Russian by J. S. Wood. 